\title{
ECX: An R Package for Studying Sensitivity of Antimicrobial Substances Using Spiral Plating Technology
}

\begin{abstract}
Gabriel Andrés Torres-Londoño and Mary Hausbeck, Department of Plant, Soil and Microbial Sciences, Michigan State University, East Lansing 48824; and Jianjun Hao, School of Food and Agriculture, University of Maine, Orono 04469
\end{abstract}

Accepted for publication 21 June 2016.

\section{ABSTRACT}

Spiral plating technique is reliable, repeatable, and more efficient than dilution plating methods in studying the efficacy of antimicrobial products. In this method, the concentration of chemicals can be varied at different positions on agar plates, but its calculation requires using a commercial software. To establish a user-friendly and costfree platform, the R package ECX was developed to calculate chemical concentrations in spiral plating technique. Mathematical models were established for calculating dispensed volume on agar plates using variables (molecular weight and agar height) that affect diffusion. In addition to the R packages, the web-based Shiny exten- sions ECX, multi, and ppm were developed to provide a graphical interface for calculating individual concentrations, multiple concentrations, and stock concentrations, respectively. No significant differences were observed $(P>0.05)$ when ECX was compared with the commercial software. The ability to import and process large datasets makes the ECX package a better option for spiral plating technique studies. Furthermore, the multiplatform nature of the ECX package overcomes limitations presented in other software. Therefore, these ECX characteristics can increase the use of the spiral plating technique for sensitivity studies.

\section{INTRODUCTION}

Chemicals such as antimicrobial substances are evaluated for their efficacy in inhibiting the growth of microorganisms by means of effective concentration (EC) and the minimal inhibitory concentration (MIC) (Förster et al. 2004; Sebaugh 2011). Depending on the purpose of the study, the effective concentration is typically defined as $50 \%$ or $90 \%$ of growth or development inhibition $\left(\mathrm{EC}_{50}\right.$ or $\left.\mathrm{EC}_{90}\right)$, or the minimum inhibitory concentration at which no inhibition occurs (Gachango et al. 2012; Hill and Schalkowsky 1990; Paton et al. 1990; Pong et al. 2010; Russell 2002; Wexler et al. 1996, 1991). Traditionally, bioassays or plate assays using pesticide-amended media have been used to determine the EC, which involves a series of chemical concentrations (minimum of 4 ) and replicated plates (Förster et al. 2004). These techniques are relatively expensive and time consuming (Wexler et al. 1996). Förster et al. (2004) found that the use of the spiral gradient dilution method using the Spiral Autoplate was an efficient substitute for traditional methods in fungicide sensitivity assays; those observations were corroborated by Gachango et al. (2012), Miles et al. (2013), Fairchild et al. (2013) Amiri et al. (2013), and others (Driever et al. 2012; Kanetis et al. 2008; Martini 2012). Several studies using this technique on human bacterial pathogens have also shown success (Hill and Schalkowsky 1990; Paton et al. 1990; Pong et al. 2010; Wexler et al. 1996, 1991). This technique has also been successfully used to evaluate the $\mathrm{EC}_{50}$ and baseline sensitivities for agricultural pesticides on different plant pathogens (Adaskaveg et al. 2011; Amiri et al. 2013; Förster et al. 2004; Gachango et al. 2012; Miles et al. 2013).

Corresponding author: Jianjun Hao. Email: jianjun.hao1@maine.edu.
The first spiral plating equipment was initially developed by Gilchrist et al. in 1973 for counting bacterial colonies (Gilchrist et al. 1973; Paton et al. 1990). In 1985, Spiral System Instruments Inc. adjusted the spiral gradient endpoint test to determine antimicrobial susceptibility of bacteria (Paton et al. 1990). The machine functions by dispensing a volume of $\sim 50 \mu \mathrm{l}$ in a gradient concentration; the deposited volume and gradient pattern is standard for this type of machines. This deposition forms an Archimedean spiral, beginning at 12 to $13 \mathrm{~mm}$ from the center of the plate, towards the edge of the plate (Fig. 1) (Förster et al. 2004; Paton et al. 1990). The deposited volume can be precisely tracked and calculated, resulting in a radially decreasing concentration gradient (Gilchrist et al. 1973; Hill \& Schalkowsky 1990; Wexler et al. 1991). The innermost loops of the spiral are omitted for evaluation, since diffusion in this area is highly affected by being next to a zero concentration area (center to dispensing starting point) (Paton et al. 1990). Förster et al. (2004) recommended removing this fungicide-free zone with a cork borer to avoid the growth of insensitive pathogens from one side of the plate to the other. Since high concentrations occur in the first laps, researchers recommend analyzing plates from radius $20 \mathrm{~mm}$ to the edge (Förster et al. 2004; Paton et al. 1990). Analysis of data collected from the spiral plate technique usually requires licensed software, such as the Spiral Gradient Endpoint (SGE) by Spiral Biotech Inc. (Wexler et al. 1996).

The EC, MIC, and total inhibition concentration (no growth of the pathogen, or TIC) can be found with the spiral plating technique by calculating the volume dispensed at the point where the desired effect is observed, and converting that volume to actual concentration of test compounds based on the stock concentration. The calculation is determined by the distance from the center of the plate to the point where these parameters are observed (Förster et al. 2004).

In addition to the distance of MIC, TIC, or EC, the calculation of the effective concentration by the commercial software includes the molecular weight (MW) of the chemical, concen- 
tration $(\mu \mathrm{g} / \mathrm{ml})$ of the stock solution, incubation time (1 or 2 days), and agar medium height (AH). The concentrations of the compound corresponding to both MIC and TIC are generated by the software SGE (Förster et al. 2004). Although the commercial software provides satisfactory calculations, its use is limited by the high cost of licensing and single-computer installation policy. To provide an alternative multi-platform application for users, our objective was to establish mathematical models based on the mechanisms of spiral plating technique, using an open-source $\mathrm{R}$ package and RStudio Shiny web applications that increase the use of the spiral plate technique for effective concentration assessment.

\section{SOFTWARE DEVELOPMENT}

Dispensing volume calculation. The radial length of each section of the template provided by the Spiral Autoplate 4000 manufacturer for counting colonies was scanned and measured with ImageJ (National Institute of Health, Bethesda, MD), and compared with the volume dispensed for each individual section by the manufacturer. The data were plotted and different mathematical models than could calculate the volumes were obtained and tested for goodness of fit using JMP 11.0 (2013, SAS Institute Inc., Cary, NC). The best fit was obtained with a four-points biexponential model. In the equation (Equation 1), volume is calculated by replacing the variable "rad," which means radius, by the distance in millimeters from the desired point to the center of the plate:

$$
i d_{1}=23.44294 \times e^{(-0.0826534 \times \mathrm{rad})}+325.32176 \times e^{(-0.237215 \times \mathrm{rad})}
$$

A quadratic model (Equation 2) was developed using the statistical software R (R Foundation for Statistical Computing, Vienna, Austria) in order to enhance the model (Equation 1) fitting. In this model, $\mathrm{id}_{1}$ is replaced by the result of the model (Equation 2):

$$
i d_{2}=-0.0538478+\left(1.0010766 \times i d_{1}\right)-\left(0.0001493 \times\left(i d_{1}\right)^{2}\right)
$$

The effect of diffusion was calculated by modeling the effect of molecular weight in the SGE output. Molecular weight was introduced into our model by using a quintic polynomial model (Equation 3).

$$
\begin{gathered}
d i f=i d_{2} \times[0.7433336+(0.0033412 \times m w)+ \\
\left(-1.21 E-0.05 \times m w^{2}\right)+\left(1.91 E-0.08 \times m w^{3}\right)+ \\
\left.\left(-1.40 E-11 \times m w^{4}\right)+\left(3.92 E-15 \times m w^{5}\right)\right]
\end{gathered}
$$

Similar to molecular weight, the linear effect of agar height (AH) and stock concentration (ppm or mg/liter), which also affect diffusion, were modeled from SGE outputs and fitted into the general model (Equation 4).

$$
\text { Concentration }=\operatorname{dif} \times(p p m / 1000) \times(3 / A H)
$$

Package building. Once the general model for dispensing was established, a series of $\mathrm{R}$ functions were developed in order to incorporate the diffusion affected by the variables of agar height, molecular weight, and concentration. Due to the open source of $\mathrm{R}$ (The R Core Team 2015), all models were packed within an R package that could be freely distributed and adopted by users. The maximum and minimum inhibitory concentrations (TIC and MIC, respectively) in the output are equivalent to tail ending radius (TER) and ending radius (ER) referred to by some commercial software (Förster et al 2004).

To fulfill the minimal structure for an R-based package, the function received distinctive names, and help and example files were created and integrated within the package. Multiplatform integrity was tested on Windows, Ubuntu, and Apple OS X.

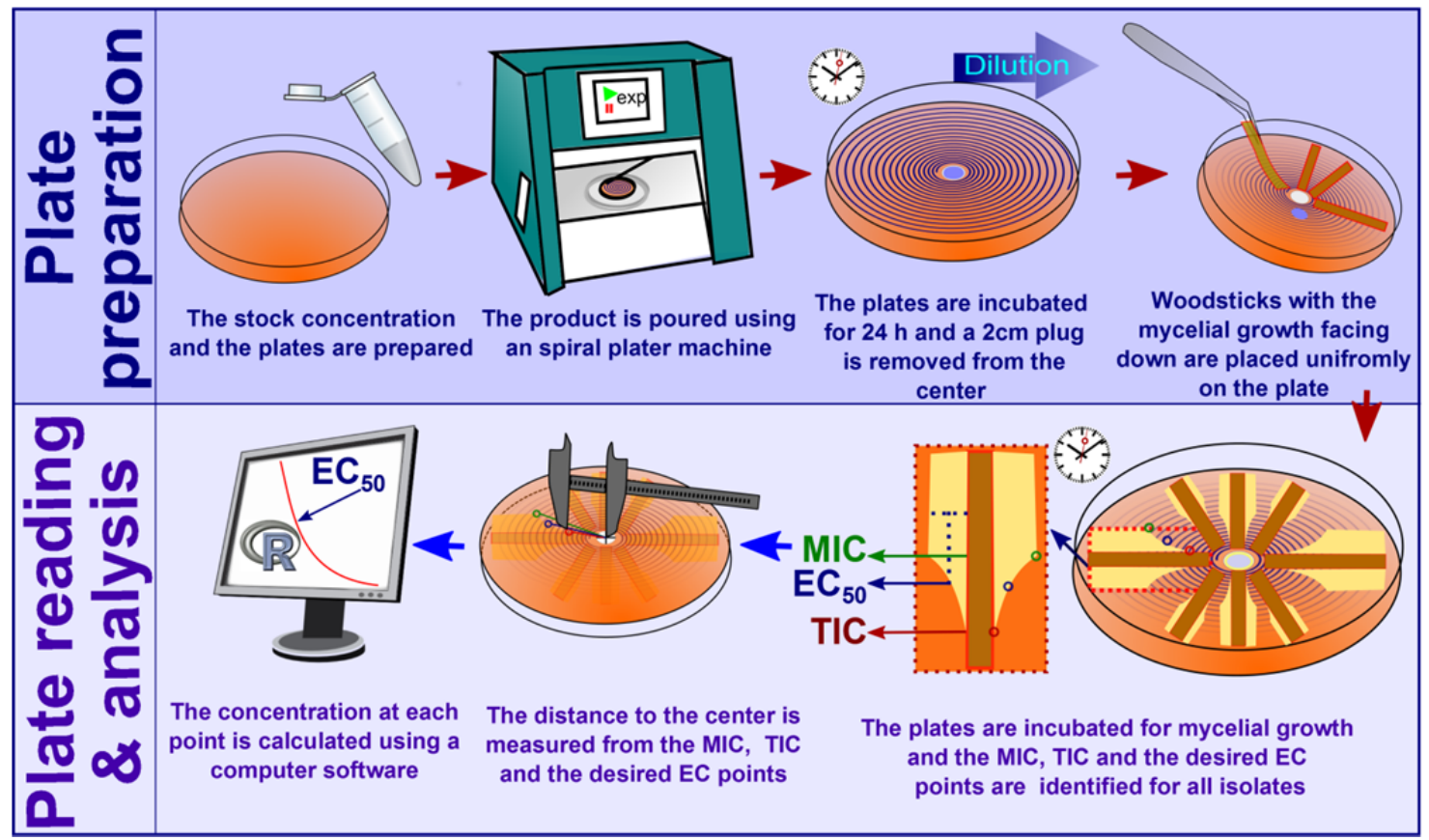

FIGURE 1

Schematic procedure for calculating chemical concentrations using spiral plate technique. 
Results of the developed package and SGE software were compared using the paired t-test, correlation, and Chi-square analyses, with the R functions t.test, cor, and chisq.test, respectively. Statistically significant differences were determined at $\alpha=$ 0.05 . Equivalence between the software was evaluated using the two one-sided tests (TOST) proposed by Robinson and Froese (2004).

Single-product and stock concentration evaluation. To calculate the dilution observed on a single spiral plate with a specific product stock concentration, the function "spcal ( )" was developed. This function calculates the dilution observed every 2 $\mathrm{mm}$ from the TIC to the MIC points when these values (rad1 and $\operatorname{rad} 2$, respectively) are input, in addition to the molecular weight (mw), stock concentration (ppm), and agar height (AH). For example, to calculate the dilution obtained between the radius 20 and $64 \mathrm{~mm}$, with a $\mathrm{EC}_{90}$ observed at $30 \mathrm{~mm}$, and the $\mathrm{mw}$, ppm, and $\mathrm{AH}$ values of 385,1000 , and 3, respectively, the calculation is accomplished using the following command:

$$
\begin{aligned}
& \text { spcal }(\operatorname{rad} 1=20, \mathrm{rad} 2=64, \mathrm{ec}=30, \mathrm{ECx}=90, \mathrm{mw}=385, \\
& \mathrm{ppm}=1000, \mathrm{AH}=3)
\end{aligned}
$$

Following the recommendation for minimum optimal value, the function was set to accept values higher or equal to $20 \mathrm{~mm}$. Similarly, the maximum value was set to $64 \mathrm{~mm}$, which is the length of the maximum radius produced by the spiral plater. Any attempt to introduce a lower or higher value than the recommended range will result in an error message.

The output of the "spcal ( )" function is presented as a data table in the R console, and it can be saved if the argument "write $=\mathrm{T}$ " is added to the command. In the Windows environment, the table can be copied to the clipboard by introducing the "copy= T" argument; the copy argument does not work under Linux nor Apple OS-X operating system environments. In addition to the table, the "spcal ( )" function produces a graphical output that includes: (i) a scheme of the growth observed in the plate with a yellow circle representing the effective concentration; (ii) a plot with the exponential dilution observed with the MIC, TIC, and EC values; and (iii) a linear regression plot with its formula (Fig. 2). This formula can be used to find values for the same product under the same conditions by replacing $X$ for the desired radius, and powering ten to the obtained result from the formula (back transformation); this reduces the dependence on R or SGE for routine analysis under specific repeated conditions. The arguments "Product" and "ECx" are merely informative and are used in the plating diagram.

\section{INDIVIDUAL AND MULTIPLE CONCENTRATIONS CALCULATOR}

The function "ECcal" was used to calculate individual or multiple concentrations obtained with the spiral gradient technique. Calculations for a single isolate are done by inputting the distance in millimeters to the center of the plate of the TIC, $\mathrm{EC}$, and MIC points individually or in a group, in addition to the parameters included for "spcal". Individual concentrations (TIC, $\mathrm{EC}$, and $\mathrm{MIC}$ ) for multiple isolates plated under the same conditions (same mw, ppm, and $\mathrm{AH}$ ), or the concentration for multiple isolates plated with different specifications can be obtained. Since many effective concentration studies require the use of several samples and the comparison of two or more antimicrobial compounds, the "ECcal" includes the possibility of import $($ write $=\mathrm{T})$ or paste $(\operatorname{Read}=\mathrm{T}$ \# Not tested on Linux $)$ data tables, when the columns headers are described (Table 1). If the data points order was mistakenly input (e.g., TIC where placed as $\mathrm{MIC}$ and vice versa) the function was programed to correct them.

The "ECcal" function enables the importation and analysis of one of the tables provided from the package by inputting the information included below. The output parameters such as saving, coping, or having additional information can be used as another option.
\# Lines with \# symbol will not run
\# Only importing
ECcal( )
\#Importing and saving
ECcal (write=T)
\#Importing and pasting into the clipboard $\mathrm{ECcal}($ Paste $=\mathrm{T})$
\#Importing and setting the intermediate sensitivity and sensitivity
\# values to 1 and $10 \mathrm{mg} /$ liter respectively
ECcal (sens $=$ T,intsens $=1$, insens $=10$ )
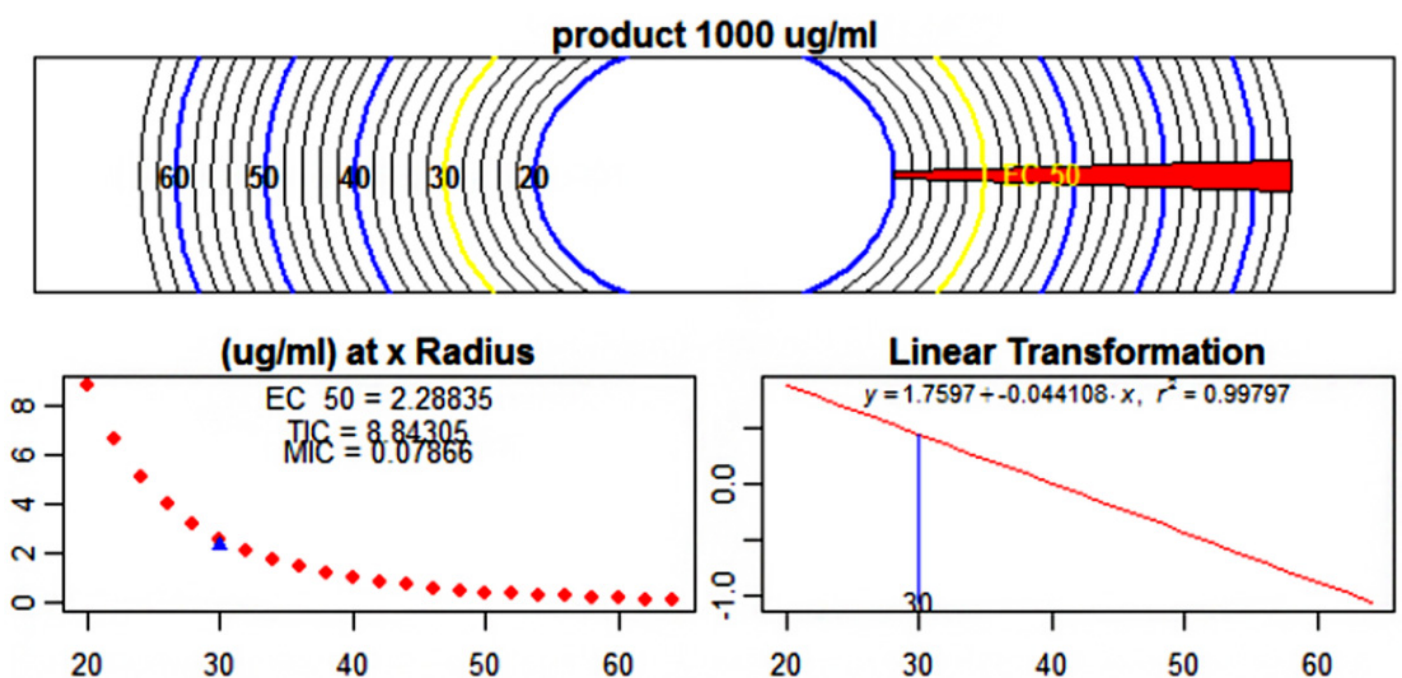

FIGURE 2

Output generated from the "spcal" function, with the parameters of $\mathrm{TIC}=20, \mathrm{TER}=64, \mathrm{EC}=30, \mathrm{ppm}=1000, \mathrm{mw}=385, \mathrm{and} \mathrm{AH}=3$. 
A single calculation can be done by manually inputting the terms. For instance, if the TIC, $\mathrm{EC}_{90}$, and MIC values of an isolate where observed at 25,35 , and $43 \mathrm{~mm}$ from the center of the plate, respectively, on a spiral plate prepared with an antimicrobial compound of $\mathrm{MW}=350 \mathrm{~g} / \mathrm{mol}$, a stock concentration of 1000 $\mathrm{mg} /$ liter, and agar height of $3 \mathrm{~mm}$, the code is:

$$
\begin{aligned}
& \mathrm{ECcal}(\mathrm{rad} 1=25, \operatorname{rad} 2=43, \mathrm{EC}=35, \mathrm{mw}=350, \mathrm{ppm}=1000, \\
& \mathrm{AH}=3)
\end{aligned}
$$

Similar to a single calculation, the values of multiple concentration with the same parameters can be analyzed manually. Suppose that the observed radius of the effective concentration for 5 isolates with the same plate description of the past example were $25,32,31,45$, and 36 . The EC for all of them can be obtained by introducing these values as "rad1" values:

$$
\text { ECcal }(\operatorname{rad} 1=c(25,32,31,45,36), \mathrm{mw}=350, \mathrm{ppm}=1000, \mathrm{AH}=3)
$$

Concentration by radius. The "Radconc" function calculates the radius at which a specific concentration is observed. For instance, to identify at which radius $3.5 \mathrm{mg} /$ liter is observed using the same parameters than the previous examples, the code is:

$$
\operatorname{Radconc}(\mathrm{Ec}=3.5, \mathrm{ppm}=1000, \mathrm{mw}=350, \mathrm{AH}=3)
$$

Stock concentration calculator for specific concentration range. To observe a specific concentration at a determined point on the plate (e.g., $5 \mathrm{mg} /$ liter at radius 35), the "ConcCAL" function can be used. The default value for the concentration distance is $42 \mathrm{~mm}$, since it is the midpoint of $150 \mathrm{~mm} \varnothing$ the plate workable area. However, it can be changed with the parameter "rad $1="$; this parameter is only required in the function if a value different from $42 \mathrm{~mm}$ is desired. The other information required by the function is the concentration desired $($ E50 $=)$, molecular weight $(\mathrm{mw}=)$, and the agar height $(\mathrm{AH})$. The code can be executed as:

$$
\begin{aligned}
& \text { \# For having } 5 \mathrm{mg} / \mathrm{liter} \text { at radius } 42 \\
& \text { ConcCAL }(\mathrm{E} 50=5, \mathrm{mw}=350, \mathrm{AH}=3) \\
& \text { \# For having } 5 \mathrm{mg} / \mathrm{liter} \text { at radius } 38 \\
& \text { ConcCAL }(\mathrm{E} 50=5, \mathrm{rad} 1=38, \mathrm{mw}=350, \mathrm{AH}=3)
\end{aligned}
$$

Web-based applications. In order to reduce the gap between $\mathrm{R}$ users and non-R users, a series of local graphical web-based applications were developed using the $\mathrm{R}$ package Shiny [install it by typing the following command in R: install.packages("shiny")] from Rstudio Inc. (Chang et al. 2015).

\begin{tabular}{|c|c|c|c|}
\hline \multicolumn{4}{|c|}{$\begin{array}{l}\text { TABLE } 1 \\
\text { Explanation of the parameters used within the function "ECcal" of the R ECX package. }\end{array}$} \\
\hline Parameterw & Expression & Data type & Description \\
\hline $\begin{array}{l}\text { Calculation } \\
\operatorname{rad} 1\end{array}$ & $\begin{array}{l}\text { Total inhibitory } \\
\text { concentration (TIC) }\end{array}$ & Numeric & Distance in $\mathrm{mm}$ from observed total inhibition point to the center of the plate \\
\hline $\operatorname{rad} 2$ & $\begin{array}{l}\text { Minimal inhibitory } \\
\text { concentration (MIC) }\end{array}$ & Numeric & Distance in mm from observed zero inhibition point to the center of the plate \\
\hline mw & Molecular Weight & Numeric & Molecular weight of the product \\
\hline ppm & $\mathrm{mg} / \mathrm{liter}$ & Numeric & Stock concentration of the product used to load the spiral plater \\
\hline $\mathrm{AH}$ & Agar height & Numeric & Agar Height. If the height is unknown, see the parameter "vol" \\
\hline vol & Volume & Numeric & If $\mathrm{AH}$ is unknown the function calculates the height if the volume used per plate is known \\
\hline \multicolumn{4}{|r|}{$\mathrm{T}^{\mathrm{T}}$} \\
\hline insens & $\begin{array}{l}\text { Insensible } \\
\text { Concentration }\end{array}$ & Numeric & Stablish the lower limit to consider a specific concentration as insensible \\
\hline intsens & $\begin{array}{l}\text { Intermediate sensible } \\
\text { concentration }\end{array}$ & Numeric & Stablish the lower limit to consider a specific concentration as intermediate insensible \\
\hline \multicolumn{4}{|c|}{ Data Management } \\
\hline info & Information & True/Falsey & Add the informative cells to the output \\
\hline Read & Read file & True/False ${ }^{\mathrm{z}}$ & Open a Window for browsing a file to be imported \\
\hline sep & Separator & $\begin{array}{l}\text { Comma ",", } \\
\text { semicolon ",", } \\
\text { or tab " } \backslash t \text { ", }\end{array}$ & $\begin{array}{l}\text { Sep is referred to the parameter used to separate the imported or pasted table. The options } \\
\text { must be entered within quotation marks }\end{array}$ \\
\hline Paste & Paste table & True/False ${ }^{z}$ & Paste a copied table to the function for being analyzed \\
\hline
\end{tabular}

The " $E C X$ " function produces the same output files than the "spcal" function. However, the function is dynamic and changes the output as the parameters are changed. In addition, the output plots and the dilution table is presented in independent tabs of the results area (Fig 3). The function is called without introducing any parameter. The functions will call the package and then a webpage will open. The code to launch this function is:

whe parameters are case sensitive and must be entered as presented on the table.

xText must be entered within quotation marks " “, for manual input.

yInformative value only. It is used exclusively if the "info" parameter is set as True.

${ }^{z}$ Bold font indicates default value of the parameter. 
\# Not introduce anything within the parenthesis ECX()

A graphical version of the "ECcal" importing function is used for previewing the table with the values to be analyzed, and to obtain the concentrations at the same time. The "multi" function runs similar to the " $E C X$ " function but the parameters input is simpler for function "multi". By browsing a file table, the type of separator used can be specified. In the table tab, the original table is loaded; while in the results tab, the TIC, EC, and MIC values are incorporated to the table (Fig 4). The table can be selected and copied. The function runs as follows:

$$
\text { multi( ) }
$$

As an additional tool to prepare the stock concentration, the function "ppm" can be used to determinate the exact product amount required to obtain a specific volume with a specific

\section{EC X Calculator}

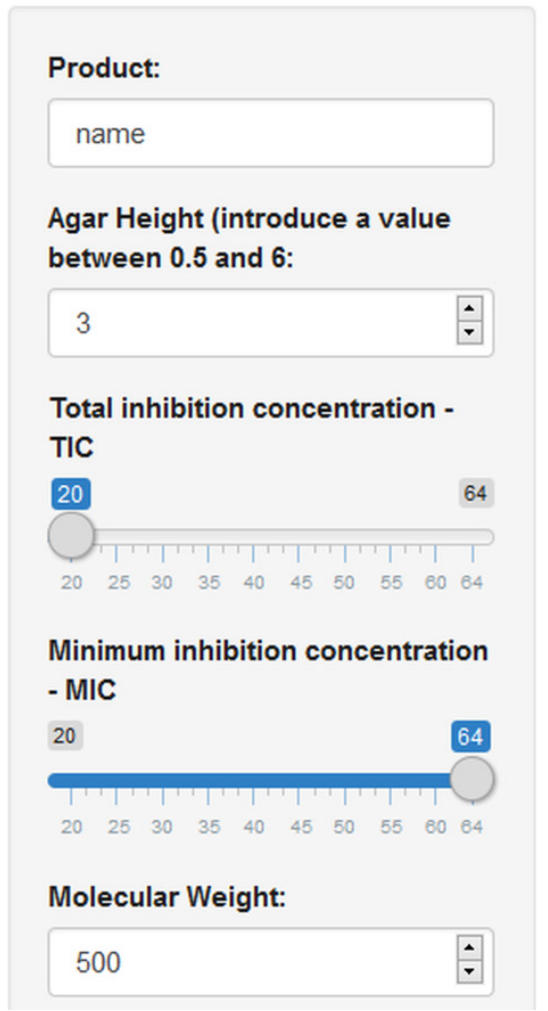

\section{Plot Table Regression Model \\ 」Download Graph}

(ug/ml)at $\mathrm{X}$ radius

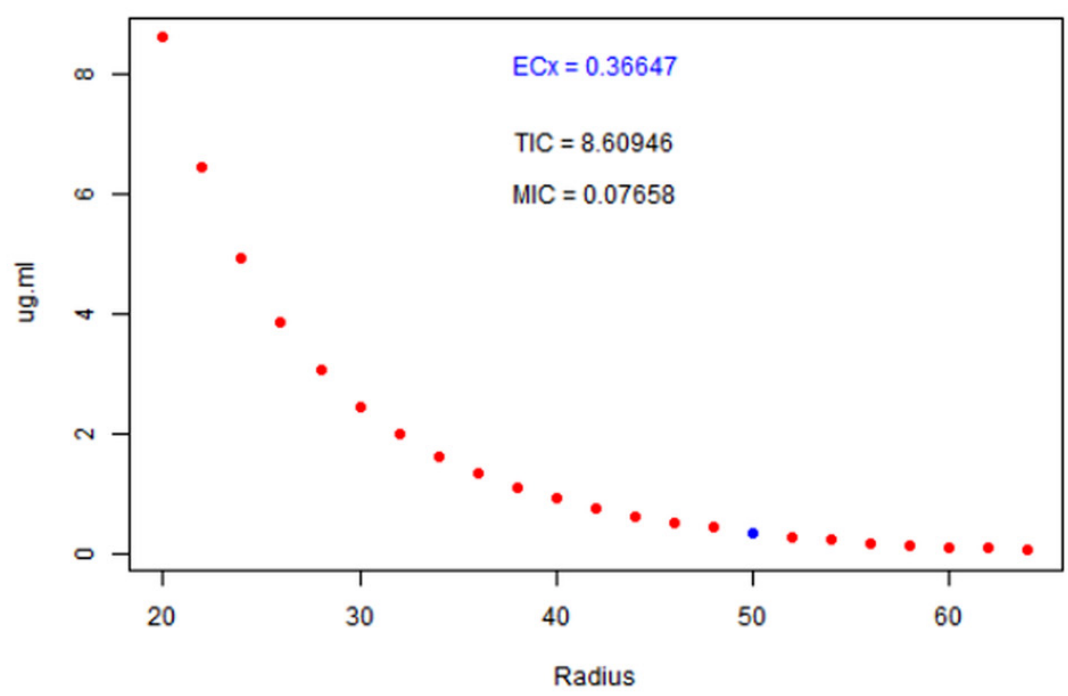

FIGURE 3

Screenshot of interface of function "ECX".

\section{EC calculator}

\section{Choose CSV File}

Choose File ...mir/test-dis coma.txt Upload complete

Separator

(-) Comma

Semicolon

Tab

\begin{tabular}{|c|c|c|c|c|c|c|c|c|}
\hline \multirow[t]{2}{*}{ Table } & \multicolumn{2}{|c|}{ Results } & & & & & & \\
\hline & Isolate & Product & mw & ppm & ah & TIC & MIC & EC \\
\hline 1 & R 19 & K & 809 & 6076 & 3.5 & 22.26972 & 1.53643 & 3.60281 \\
\hline 2 & P 16 & $\mathrm{P}$ & 159 & 8887 & 0.5 & 166.01669 & 4.74931 & 10.82847 \\
\hline 3 & Y 5 & $\mathrm{~T}$ & 407 & 6957 & 2.5 & 5.011 & 3.44041 & 5.011 \\
\hline 4 & J 13 & 0 & 952 & 883 & 1.5 & 14.89721 & 0.13252 & 0.24948 \\
\hline 5 & R 11 & $\mathrm{~J}$ & 151 & 8631 & 3.5 & 16.59176 & 1.34169 & 3.30309 \\
\hline 6 & G 5 & $\mathrm{R}$ & 770 & 9692 & 2.5 & 3.78347 & 1.29362 & 2.80715 \\
\hline 7 & C 11 & $\mathrm{~L}$ & 179 & 1153 & 5.5 & 0.34504 & 0.15911 & 0.28593 \\
\hline
\end{tabular}


concentration of the product ( $\mathrm{mg} /$ liter or $\mu \mathrm{l} /$ liter). The function requires the name of the product, percentage of active ingredient, product presentation (liquid or solid), desired final concentration ( $\mathrm{mg} / \mathrm{liter}$ ), and desired final volume in $\mathrm{ml}$. The output is a text indicating the required amount of product necessary to obtain the final volume at the desired concentration (Fig 5). The calculator is launched by the code:

$$
\text { ppm( ) }
$$

In some $\mathrm{R}$ environments when the webpages are closed, $\mathrm{R}$ console appears like "Browse[1]>" and users are required to stop its running by pressing the escape key.

Supporting files. A series of tables with 10,000 randomly created data points were prepared as an example for running the "ECcal" function (https://github.com/GabrielTorres/ECX/releases/tag/3.01). The columns of all of them, except "test-dis coma.txt", were separated by a tab space (sep = "|t"). The files include a complete table with TIC, MIC, and EC values. Tables with only TIC and MIC values, and with only EC values were also included. The tables with TIC, $\mathrm{EC}$, and MIC ordered (RC_ord); TIC, EC, and MIC disordered (RC_disord); with TIC and MIC (RC_2v); and EC only (RC_sv) were incorporated within the package and can be called and tested as follows:

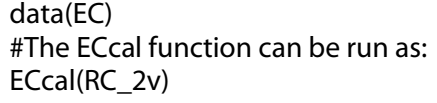

\section{MAIN FINDINGS}

We have developed an R-based package and a web-based extension freeware to calculate effective concentrations of antimicrobial compounds. Results obtained with this new package were similar to those obtained with the commercial SGE software. The results from this cost-free packages were reliable and reproducible. The user-friendly interface, the ability to import datasets from other software and the multi-platform nature of those packages provide extra tools for broad and powerful analyses.

Spiral gradient endpoint techniques are precise and costeffective compared to traditional dilution methods (Förster et al. 2004; Paton et al. 1990; Pong et al. 2010). As Hill and Schalkowsky (1990) indicated, we found MW is the main component to explain diffusion. The effectiveness of the diffusion adjustment to the SGE formula done by Hill and Schalkowsky was validated by Wexler et al. (1996). For plant pathogens, Föster et al. (2004) found that the 1-day and 2-day parameters designed for aerobic and anaerobic bacteria, respectively, were adequate to calculate the $\mathrm{EC}_{50}$ value for conidial germination and mycelial growth, specifically. The present study focused on mycelial growth; results were similar to those obtained in the commercial software when the "day" option is set to 2 .

In the R package ECX, concentration was expressed as "ppm" and " $\mathrm{g} / \mathrm{ml}$ " for variables and outputs, respectively. Users can define any other units, as long as they are kept consistent. SGE exclusively calculates the concentration at the specified TER and ER points. In contrast, the Conc function and the Shiny web application in ECX calculate the concentrations for the range between TER and ER in 2-mm increments. The output of those functions also provides the linear regression formula of the linear transformation. This formula can be used to find values for the same product under the same conditions by replacing $\mathrm{X}$ for the desired radius, and powering ten to the obtained result from the formula (back transformation); this reduces the dependence on $\mathrm{R}$ or SGE for routine analysis under specific repeated conditions.

The ECX package will be available at: https:/github.com/GabrielTorres/ECX. While the objective of these new packages is not to be a substitute for the commercial software, its open-source characteristics, wide range of data that can be manipulated under a single analysis process, and relatively user-friendly interface can enhance the use of spiral autoplate for antimicrobial compound analysis.

\section{ppm calculator}

Product name:
Product
Percentage A.I.
100
State of the A.I.
Liquid
Solid
Desired concentration in mg/liter
100
Desired final volume in $\mathrm{ml}$
1000

To obtain 1 liters of Product at $100 \mathrm{mg} /$ liter, mix $100 \mathrm{ul}$ of Product and set the final volume to $1000 \mathrm{ml}$

\section{FIGURE 5}

Screenshot of the output of function "ppm". 


\section{CONCLUSION}

A series of functions for calculating the concentration deposited by spiral plater machines were developed. All individual functions presented results similar to SGE (Table 2). The Shiny ECX application had the same performance as the spcal function package (Fig 6). The Shiny ppm extension was also tested and proven to be accurate when the concentrations of commercial diluted products were calculated. The result displays the number of grams, milligrams, milliliters, or microliters that the user must add to the final volume.

There were no significant differences between the SGE software and the R package when the output of a mock dataset of 3435 data points was evaluated at $P=0.95$. The Chi square and the $t$-test produced $P$ values of 1 and 0.88 , respectively, at $\alpha=$ 0.05. The correlation was higher than $99.8 \%$.

\section{ACKNOWLEDGMENTS}

The authors express their gratitude to Dr. William Kirk, Qianwei Jiang, and Paula Somohan for the software testing and feedback, as well as Dr. Andrew Robinson for his comments on the equivalence test analysis.

Funding for the present study came from Dr. Mary Hausbeck's laboratory at Michigan State University.

\begin{tabular}{|c|c|c|c|}
\hline \multicolumn{4}{|c|}{$\begin{array}{l}\text { TABLE } 2 \\
\text { Concentrations calculated at radius } 30 \mathrm{~mm} \text { for different } \\
\text { antimicrobial compounds (ppm }=650 \mathrm{mg} / \mathrm{liter}, \mathrm{AH}=3 \mathrm{~mm} \text { ). }\end{array}$} \\
\hline Product & $\begin{array}{c}\text { Molecular } \\
\text { weight }\end{array}$ & SGE & ECX \\
\hline Amikacin & 586 & 1.77 & 1.83 \\
\hline Control & 1000 & 1.74 & 1.82 \\
\hline Erythromycin & 734 & 1.74 & 1.81 \\
\hline Fluopicolide & 385 & 1.91 & 1.91 \\
\hline Mefenoxam & 279 & 2.04 & 1.94 \\
\hline Metronidazole & 171 & 2.19 & 1.89 \\
\hline Nalidixic acid & 232 & 2.11 & 1.93 \\
\hline Prothioconazole & 480 & 1.83 & 1.86 \\
\hline Propamocarb hydrochloride & 188 & 2.17 & 1.91 \\
\hline Rifampicin & 823 & 1.73 & 1.82 \\
\hline Thiabendazole & 201 & 2.15 & 1.92 \\
\hline Topsin & 342 & 1.96 & 1.92 \\
\hline Tobramycin & 468 & 1.84 & 1.87 \\
\hline Zoxamide & 337 & 1.96 & 1.93 \\
\hline
\end{tabular}

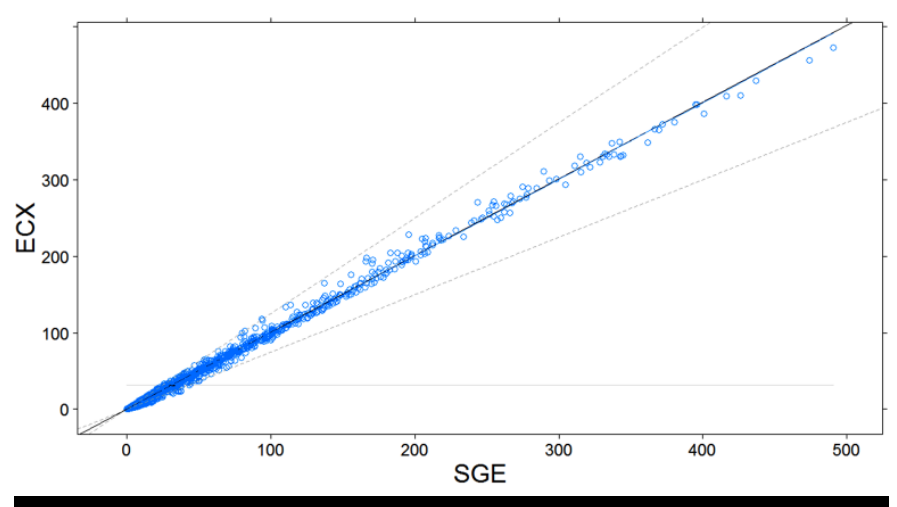

FIGURE 6

Equivalence test (TOST) between the values obtained with the commercial software SGE and the values estimated with ECX.

\section{LITERATURE CITED}

Adaskaveg, J. E., Förster, H., and Wade, M. L. 2011. Effectiveness of kasugamycin against Erwinia amylovora and its potential use for managing fire blight of pear. Plant Dis. 95:448-454.

Amiri, A., Heath, S. M., and Peres, N. A. 2013. Phenotypic characterization of multifungicide resistance in Botrytis cinerea isolates from strawberry fields in Florida. Plant Dis. 97:393-401.

Chang, W., Cheng, J., Allaire, J. J., Xie, Y., and McPherson, J. 2015. Shiny: Web application framework for R. RStudio Inc., Boston, MA. http://www.rstudio.com

Driever, G. F., Förster, H., Thompson, D. C., and Adaskaveg, J. E. 2012. Toxicity and resistance potential of selected fungicides to Galactomyces and Penicillium spp. causing postharvest fruit decays of citrus and other crops. Plant Dis. 96:87-96.

Fairchild, K. L., Miles, T. D., and Wharton, P. S. 2013. Assessing fungicide resistance in populations of Alternaria in Idaho potato fields. Crop Prot. 49:31-39.

Förster, H., Kanetis, L., and Adaskaveg, J. E. 2004. Spiral gradient dilution, a rapid method for determining growth responses and $50 \%$ effective concentration values in fungus-fungicide interactions. Phytopathology 94:163-170.

Gachango, E., Hanson, L. E., Rojas, A., Hao, J. J., and Kirk, W. 2012. Fusarium spp. causing dry rot of seed potato tubers in Michigan and their sensitivity to fungicides. Plant Dis. 96:1767-1774.

Gilchrist, J. E., Campbell, J. E., Donnelly, C. B., Peeler, J. T., and Delaney, J. M. 1973. Spiral plate method for bacterial determination. Appl. Microbiol. 25:244-252.

Hill, G. B., and Schalkowsky, S. 1990. Susceptibility testing of antimicrobial agents: development and evaluation of the spiral gradient endpoint method for susceptibility testing of anaerobic Gram-negative bacilli. Rev. Infect. Dis. 12:S200-S209.

Kanetis, L., Förster, H., and Adaskaveg, J. E. 2008. Baseline sensitivities for new postharvest fungicides against Penicillium spp. on citrus and multiple resistance evaluations in P. digitatum. Plant Dis. 92:301-310.

Martini, C. 2012. Phenotype and genotype characterization of Monilinia spp. isolates and preformed antifungal compounds in peach peel fruit at different developmental stages. PhD. thesis. Univ. of Bologna, Italy.

Miles, T. D., Miles, L. A., Fairchild, K. L., and Wharton, P. S. 2013. Screening and characterization of resistance to succinate dehydrogenase inhibitors in Alternaria solani. Plant Pathol. 63:1-10.

Paton, J. H., Holt, H. A., and Bywater, M. J. 1990. Measurement of MICs of antibacterial agents by spiral gradient endpoint compared with conventional dilution method. Int. J. Exp. Clin. Chemother. 3:31-38.

Pong, R., Boost, M. V., O’Donoghue, M. M., and Appelbaum, P. C. 2010. Spiral gradient endpoint susceptibility testing: A fresh look at a neglected technique. J. Antimicrob. Chemother. 65:1959-1963.

R Core Team. 2015. R: A Language and Environment for Statistical Computing. R Foundation for Statistical Computing, Vienna, Austria.

Robinson, A. P., and Froese, R. E. 2004. Model validation using equivalence tests. Ecol. Modell. 176:349-358.

Russell, P. E. 2002. Sensitivity baselines in fungicide resistance research and management. Crop Life Int., Brussels, Belgium.

Sebaugh, J. L. 2011. Guidelines for accurate EC50/IC50 estimation. Pharm. Stat. 10:128-134.

Wexler, H. M., Molitoris, E., Jashnian, F., and Finegold, S. M. 1991. Comparison of spiral gradient and conventional agar dilution for susceptibility testing of anaerobic bacteria. Antimicrob. Agents Chemother. 35:1196-1202.

Wexler, H. M., Molitoris, E., Murray, P. R., Washington, J., Zabransky, R. J., Edelstein, P. H., and Finegold, S. M. 1996. Comparison of spiral gradient endpoint and agar dilution methods for susceptibility testing of anaerobic bacteria : A multilaboratory collaborative evaluation. J. Clin. Microbiol. 34:170-174. 